\title{
Vom strahlenden Leben ohne Sachverstand
}

\author{
O. Fluri
}

1 Egger N, Fluri O, Geiger P. Die Entwicklung von normalen und cortisonbehandelten Ratten unter besonderer Berücksichtigung der Myelinisierung. Dissertation. Universität Bern, nicht publiziert.

2 Trueb PR (Hrsg.). Kompendium für ärztliche StrahlenschutzSachverständige. Bern: Verlag Paul Haupt; 2002. S. 80/136/138

3 www.hospvd.ch/public/instituts/ ira/enquete/eng/survey.htm.
Seit ich im Kindergartenalter im Schuhgeschäft im strahlenden Durchleuchtungsapparat mit meinem Hallux spielte und meine Mutter mit strahlenden Augen bat, sie möchte doch bald wieder Schuhe kaufen, hat sich im Strahlenschutz zu Recht einiges getan. Es sind aber doch zwei verschiedene Paar Schuhe, wenn ich nach 22 Jahren Betreiben einer Praxisröntgenanlage meinen Sachverstand prüfen lassen muss, wie wenn ich morgen eine einrichten würde.

Der Sachverstand ist in unserem Land eine willkürliche Grösse. Bisher genügten 5 Jahresstunden Physik fürs erste Prope, die Strahlenschutzthemen im Rahmen der Radiologievorlesung und die Kenntnisse der biologischen Effekte aus der Vorlesung für Strahlentherapie, um unseren Strahlenverstand zu schärfen. Diese Themen wurden auch geprüft - sehr zum Leidwesen der A- und B-Maturanden, die mit Physik kämpften, während ich mit der C-Matur für die Ergänzungsprüfung in Latein büffelte. Irgendein Numerus clausus musste einem ja das Leben versauern.

Bei unserer Gruppendissertation [1] kontrollierten wir die Stoffwechselkinetik der Myelinscheiden unter Einfluss von Cortison mit Radioisotopen und erhielten signifikante Resultate unter Benützung eines Szintillationsspektrometers und des Sachverstands.

Vor etwa 20 Jahren wählte mich der Regierungsrat des Kantons Solothurn zum Experten für Chemie und Biologie am Wirtschaftsgymnasium der Kantonsschule Solothurn. Von Hunderten von Maturanden hörte oder las ich Richtiges und Falsches vom Bohrschen Atommodell bis zur Röntgenröhre und zu strahlenbedingten Chromosomenaberrationen. Beim Trennen von Spreu und Weizen ergaben sich keine Sachverstandsdifferenzen zwischen Lehrer und Experten. Mein Sachverstand wurde zwar mit dem Expertenhonorar von 30 Franken pro Stunde oder 8 Franken pro schriftliche Arbeit nicht allzu hoch eingestuft. Immerhin bekam der Physikexperte nicht mehr.

1986 WK mit der Spit Abt 61 als Facharzt für Allgemeinmedizin FMH. Das Basisspital im Bunker wurde betriebsbereit gemacht. Auch das Röntgen musste laufen. Ich wurde Röntgenchef. Die Röntgenanlage war noch nicht «eingeschossen». Keine Belichtungstabelle, keine Filme, keine Chemikalien, der Entwicklungsautomat noch nie gelaufen. Vorhanden war nur die Bewilligung des BAG, die mich als einzigen Röntgenverständigen der Spitalabteilung auswies Verstand nach Bedarf! - und der Datenschutz lässt grüssen. Am Ende des WK konnte der Abteilungsadjutant seine massive Spondylose L5/S1 dokumentiert nach Hause tragen und der Berufsfotograf, dem ich im Einzelunterricht mühsam die Einstelltechnik beibrachte, meldete sich ab - es war sein letzter WK.

1979 habe ich meine Praxis eröffnet. Die Röntgeneinrichtung entstand durch «learning by doing» zusammen mit Fachleuten von Firmen, die damals beständiger waren als heute. Vor allem Herr Laubscher - seine Konstanzprüfungskassette wird im Strahlenschutzbuch auf Seite 80 [2] erwähnt - unterstützte mich im «Einzelunterricht», bis alles klappte. Seither machen wir Röntgenbilder, welche die Beurteilungskriterien erfüllen, im Gegensatz zu einigen Bildbeispielen im Strahlenschutzbuch. Es gibt natürlich auch bei uns Ausreisser, aber wir publizieren sie nicht.

Die Tatsache, dass nach all diesen Begegnungen und Erlebnissen mit Strahlenphysik nun eine Prüfung ansteht, irritiert wohl nicht nur mich, sondern zahllose Ärzte mit Praxisröntgen und zunehmend ergrauenden Haaren im Alter über 45 Jahren. Wie denkt ihr darüber?

Die Notwendigkeit dieser Übung wird durch das BAG mit der Strahlenexposition der Bevölkerung begründet. Im Strahlenschutzbuch [2] werden dazu unglaubliche Behauptungen aufgestellt: In der Schweiz würden pro Jahr pro 1000 Einwohner 1360 Röntgenuntersuchungen durchgeführt. Die Röntgenfrequenz in meiner Praxis beträgt 268 Aufnahmen pro tausend Patienten pro Jahr (absolut 322 Aufnahmen bei 1200 Patienten im Jahr 2001). Gemäss einer Studie von F. Bossard, Thun, macht ein Allgemeinpraktiker im Schnitt 373 Röntgenuntersuchungen pro Jahr [3]. Das entspricht 20-30\%!

Daneben schicke ich pro Arbeitstag 0-2 Patienten ins Spital zum Röntgen. Dabei handelt es sich aber um Patienten, und wir wissen, dass längst nicht jeder Einwohner Patient ist.

Bei der Betrachtung der Tabelle über die Schätzungen der Kollektivdosis wird das Ganze noch absurder. 470 Schädelröntgen pro 1000 Einwohner sollen pro Jahr durchgeführt werden. Ich selber trage dazu 11,5 Aufnahmen bei und 
verlange bei den meisten Schädelhirnabklärungen im Spital ein MRI.

Ich glaube nicht, dass ich in meiner Praxis ein Röntgenexot bin. Eher glaube ich, dass sich die Autoren gründlich irren, wie sie auch grosszügig mit Sievert, Millisievert und sonstigen Zehnerpotenzen umgehen.

Die Frage ist wohl berechtigt, welche Fehler und Irrtümer wir lernen müssen, um diese Prüfung zu bestehen.
Auf diese Prüfung ist meiner Ansicht nach zu verzichten. Sie ändert nichts an meinem bisherigen Röntgenverhalten. Die Notwendigkeit wurde in obstrusen Dunkelkammern ausgebrütet und mit unplausiblem Zahlenmaterial begründet.

Warum haben unsere Vertreter der FMH, der SGAM und das KHM das akzeptiert? 